\title{
An inflammatory assemblage
}

\author{
The multiprotein inflammasome complexes are important in responses to microbes but are also increasingly \\ recognized as having key pathogenic roles in a variety of diseases from cancer to obesity.
}

ten nflammation is a fundamental response of the innate immune system to noxious stimuli. It has become somewhat trite to say that the past two decades have seen an explosion of interest in innate immunity, but progress in this area has been nothing if not revolutionary, as was emphatically demonstrated by last year's Nobel prize. Another 'minirevolution' is taking place in the field of inflammasome research. As the name suggests, inflammasomes are multimolecular complexes that assemble into a platform for the activation of proinflammatory caspase-1. This definition includes the Nod-like receptor (NLR) proteins NLRP1, NLRP3, NLRC4, NLRP6 and Naip5, as well as the DNA-sensing complex of AIM2. Inflammasome-activated caspase-1 is then used for activation of the proinflammatory cytokines interleukin $1 \beta$ (IL-1 $\beta$ ) and IL-18. It seems certain that additional inflammasomes will be described in the near future.

There is increasing appreciation that the various 'flavors' of inflammasomes impinge on a diverse array of inflammatory processes, and the rapid progress in this area has inspired this focus issue of Nature Immunology (http://www.nature.com/ni/focus/inflammasomes). The fives pieces that make up this focus cover most of the key aspects of inflammasome biology, including pathogen recognition, activation and regulation, as well as an evolving story indicating their involvement in many metabolic conditions. This diversity of inflammasome function is summarized by Richard Flavell and colleagues in their focus overview.

A key function of the inflammasome is surely the defense against pathogenic microbes; this forms the topic of the review by Gabriel Núñez and colleagues. NLRC4 is an exemplar of the protective role of inflammasomes. Together with Naip5, NLRC4 recognizes motifs associated with pathogenic bacteria, which results in IL-1 $\beta$ - and/or IL-18-dependent clearance of bacteria. Another important outcome of NLRC4 activation is the induction of pyroptosis in macrophages that serve as hosts to pathogenic bacteria such as Salmonella. This not only robs macrophage-tropic bacteria of their niche, it also exposes them to the extracellular world and the full onslaught of the immune system. The messy nature of pyroptosis is also an effective means of disseminating the inflammatory response and alerting the host to the presence of pathogens. Other inflammasomes, especially NLRP3, are involved in the response to pathogens, and it seems likely that additional members and functions will be identified in the coming years.

IL- $1 \beta$ is the original 'endogenous pyrogen', so all this inflammasome activity must be tightly regulated to avoid potentially harmful inflammation. Indeed, one of the most exciting results to emerge from this area has been the finding that certain heritable autoinflammatory conditions are associated with gain-of-function mutations in the gene encoding NLRP3. IL-1 $\beta$ is the key thread that connects all these condi- tions, because their pathogenesis can be ameliorated by antagonism of IL-1 $\beta$. In their review, Kate Fitzgerald and colleagues explore inflammasome activation and the regulatory mechanisms that have evolved to keep inflammasome activity in check. Among all the inflammasomes, NLRP3 seems to be unique in its promiscuous ability to be activated by a wide array of unrelated stimuli, including microbe- or host-derived molecules and even totally inorganic entities such as silica, asbestos and, most famously, alum. Although models have been put forward to explain how this wanton activation can occur, a watertight unifying mechanism remains elusive.

Guido Kroemer and colleagues discuss another promising area of inflammasome research: the involvement of inflammasomes in carcinogenesis. Here the inflammasome seems to have an influential but confusing role. Inflammasome-mediated release of IL-1 $\beta$ can drive the chronic inflammation that leads to cancer, but inflammasome-triggered pyroptosis of cancer cells can also help in their elimination. The literature abounds with such apparently contradictory effects. An important corollary of this is that modulating inflammasome activity may result in unexpected and harmful side effects through the promotion of tumorigenesis. This complexity still needs to be resolved, but it seems likely that the outcome of inflammasome activity in cancer depends on the nature of the activation trigger, the particular inflammasome in question and the tissue location.

Jenny Ting and colleagues explore the final topic of the focus issue: the involvement of the NLRP3 inflammasome in metabolic diseases. Obesity and type 2 diabetes are an emerging scourge of the developed world, and chronic inflammation is increasingly thought to be an important etiological component of these diseases. A breakthrough in this area was provided by independent studies showing that a greater abundance of saturated fats or cholesterol crystals can activate the NLRP3 inflammasome. Gout, the so-called 'disease of kings', arises from an accumulation of dietary purines and, similarly, results in the activation of NLRP3. Interestingly, all these diseases are amenable to control by direct antagonism of IL-1 $\beta$. Other inflammasome triggers, both endogenous and exogenous, will probably emerge in the coming years and will be found to influence the manifestation of metabolic diseases. As for metabolic diseases, the gut will almost certainly be a key area of focus for future studies. Not only is the gut the site of nutrient absorption but also the resident microbial ecosystem of the gut may fine tune the threshold of inflammation at the level of NLRP3.

It seems clear that inflammasomes will be a productive area of research in the coming years. Delineating the mechanisms and consequences of their activation may provide a link with which to connect a host of otherwise unrelated responses and conditions. 